\title{
Update on the Oversight of Animal Care and Use Programs
}

\author{
A recap of a recent NABR seminar held at the 69th AALAS Meeting in Baltimore.
}

\section{B. Taylor Bennett and Matthew R. Bailey}

ach year at the national meeting of the

American Associations for Laboratory Animal Science (AALAS), the National Association for Biomedical Research (NABR) organizes a seminar where representatives from the United States Department of Agriculture (USDA), the National Institutes of Health Office of Laboratory Animal Welfare (OLAW) and AAALAC International provide an update of their organizations' oversight programs. This article will highlight some of the questions posed to the USDA and OLAW and the answers provided by the respective agency's representatives, Dr. Elizabeth Goldentyer and Dr. Patricia Brown.

\section{Responses from USDA}

The USDA was asked for an explanation of the "annual focused inspection" process. While the agency is required by language in the Animal Welfare Act (AWA) to inspect each research facility (RF) annually, they do have flexibility in how 'in-depth' the inspection needs to be. Based on risk factors such as history of compliance, some RFs could be subject to a "focused" rather than full routine inspection. During a focused inspection, the VMOs will still look at the animals and check some records, but they will not necessarily cover every area of the facility and the number of protocols they would for a full inspection. Based on their observations, VMOs always have the prerogative of conducting a full inspection.

Another question to the USDA involved the reporting of animals that received anesthesia for restraint in order to perform procedures involving only momentary or transient pain, such as obtaining a blood sample, or that involve no pain, such as an imaging procedure. The USDA responded that if the purpose of the anesthesia is to assure safe handling, then the animal should be reported in Column $\mathrm{C}$ of the annual report; if the purpose is to minimize pain associated with the procedure, then the animal would be reported in Column D. Animals that are being held but not used for research but that undergo anesthesia for restraint as part of routine veterinary or colony management should still be reported in Column B.
The USDA representative also addressed whether all research facilities that sell animals are required to have a license. The term "dealer" is defined in the Animal Welfare Act as "any person who, in commerce, for compensation or profit, transports buys or sells..." A state institution is not considered a person and therefore does not need a license.

\section{Responses from OLAW}

There was a question to OLAW regarding the reporting of an incident at an institution with a Public Health Service (PHS) Assurance when the incident did not involve animals covered by the assurance. The OLAW representative responded that if the event was programmatic in nature (i.e., affecting the entire facility) then it must be reported. If the incident was localized to a single room or area that does not contain animals funded by PHS, the National Science Foundation or the Department of Health and Human Services, then it does not need to be reported.

OLAW was also asked for its position on counting vertebrate animals at or around birth. The OLAW representative indicated that neonatal rodents should be accounted for when they are first manipulated, such as during the first cage change or at genotyping. Zebrafish are considered live vertebrate animals at 3 days post fertilization and should be accounted for at or around that time. PHS Policy ${ }^{1}$ applies to all live vertebrate animals and requires institutions to establish mechanisms for documenting and monitoring the approximate number of animals acquired and produced, whether or not they meet the criteria to be used for a specific research purpose. Observing litters and hatchlings early also allows for tracking of mortality rates, which is important for monitoring the health of colonies, especially of genetically manipulated animals.

Finally, OLAW was asked to define what is meant by a location that is not part of the animal care and use program overseen by IACUC. Under PHS Policy, the Animal Welfare Assurance must include "a list of every branch and major component of the institution, as well as a list of every branch and major component of any other institution, which is to be included under the Assurance." 1,2 Based on this language, any location contained within the facilities of a branch or major component as described in the assurance would be under IACUC oversight. Any other location would not. Concerning facilities that are covered, OLAW clarified that laboratories where animals are briefly kept for procedures such as dosing or weighing do not need to be visited by the IACUC during the semiannual inspection. Areas where any form of surgical manipulations are conducted (including minor, major, survival, or non-survival surgeries) or locations where animals are held for more than 24 hours do need to be inspected. All animal housing and performance areas need initial IACUC approval. Moving animals to new IACUC-approved space is not considered a significant change and can be tracked administratively. The IACUC is still responsible for general oversight of all areas.

It is important for those involved in the management of an institution's animal care and use program to stay informed on current issues and trends in the multidimensional oversight process in place in the US. In this article, we have addressed several questions that were presented recently to US oversight agency representatives, covering new issues as well as ones that have been frequently discussed but bear repeating.

\section{B. Taylor Bennett* and Matthew R. Bailey National Association for Biomedical Research, Washington, DC, USA. \\ *e-mail:btbdvm@yahoo.com}

Published online: 4 February 2019 https://doi.org/10.1038/s41684-019-0244-7

\footnotetext{
References

1. Public Health Service. Policy on Humane Care and Use of Laboratory Animals (US Department of Health and Human Services, Bethesda, MD, 1986, revised 2015). https://olaw.nih.gov/ policies-laws/phs-policy.htm

2. Office of Laboratory Animal Welfare, National Institutes of Health 2018. OLAW Guidance, Obtaining an Assurance. Found on line at: https://olaw.nih.gov/guidance/obtaining-an-assurance.htm.
} 